East African Journal of Science, Technology and Innovation, Vol. 2 (4): September 2021

This article is licensed under a Creative Commons license, Attribution 4.0 International (CC BY 4.0)

\title{
Factors influencing Euryarchaeal gut methanogens distribution in dairy cattle in smallholding farms
}

\author{
1NGETICH D K., 1BETT R C., ${ }^{1}$ GACHUIRI C K., 1KIBEGWA F M. \\ 1*Department of Animal Production, University of Nairobi, College of Agriculture and Veterinary Sciences, P.O. BOX 29053, - \\ 00625, KANGEMI- KENYA. \\ *Corresponding author: ngetichkipkorirdenis@gmail.com
}

\begin{abstract}
Guts of ruminants contain symbiotic domains (Eubacteria, Archaea and Eukarya) that aid in the breakdown of consumed carbohydrates from plants to simple molecules that can be absorbed into the ruminant's bloodstream. Methanogenesis occurs during the gut fermentation and methane gas is released in the final step of biomass degradation from the fermentation chambers. The Archaea that play a major role critical for methane emissions are methanogens and are found freely in the ruminants' gut. Methane production from ruminants has attracted global attention due to their input on the Green House Gases effect, contribution to global warming and negative effects on farmers' productivity. The objective of this study was to determine the factors contributing to the methanogens' gut distribution in dairy cows from smallholder farms using next generation sequencing techniques. A total of 48 samples from smallholding dairy farms were used during this study and were collected from Kenya (Kiambu county) and Tanzania (Lushoto and Rungwe). The collected data samples from the experimental animals were from both the rumen fluid (6) and fecal (42). Samples were analyzed using metagenomic approaches and statistical analysis was undertaken using IBM SPSS statistics software version 28.0.0.0. Results showed that the gut site along the gastrointestinal tract and the feeding regime significantly contributed to the distribution and presence of various methanogenic species $(\mathrm{P}<0.1)$. The herd and the genotype had no statistical effect. A total of 12 families were identified. The family Methanobacteriaceae was identified with the leading number (8) of the methanogenic species. A third of the identified families showed presence for at least two methanogenic species with Methanobrevibacter ruminantium being abundant. For proper curbing mechanisms, efforts to reduce methane release should be channeled to the whole gastrointestinal tract and advanced studies carried out on any potential interspecies presence facilitation and/or elimination.
\end{abstract}

Keywords: Archaea; Methane; rectum; rumen; species

Cite as: Ngetich et al., (2021 Factors influencing Euryarchaeal gut methanogens distribution in dairy cattle in smallholding farms. East African Journal of Science, Technology and Innovation 2(4).

\section{Introduction}

Ruminants have a capability of converting low quality and/or high fibre feeds through the fore stomach's fermentation to high valued human products. Ruminants remove their end products of digestion/ fermentation through absorption,
Received: $\quad 17 / 06 / 21$

Accepted: $\quad 11 / 08 / 21$

Published: 25/09/21 eructation or passage. After nutrients absorption, domesticated species give man suitable consumable outputs; such as milk, meat and other non-consumable products such as manure, traction and capital assets. Guts of ruminants 
contains symbiotic domains (Eubacteria, Archaea and Eukarya) that aid in breakdown of consumed cellulose and other carbohydrates from plants to simple bits for absorption into the ruminant's bloodstream that otherwise mono-gastric classes would not have easily utilized (Liu et al., 2017). With references to older studies and literature, Archaea was placed in the domain of bacteria. It was later discovered beyond any reasonable doubt that it was unique and different from bacteria. With time, two initial super phyla for Archaea were discovered; Euryarchaeota and Crenarchaeota (Baker et al., 2016; Rinke et al., 2013). This initial division was arrived at because of their vast differences in temperature requirements, physiological state, metabolic preferences and their type of primitive forms though were noted to conserve genetic cores despite their variation that made them retained in the unique category. Collectively, they were thought to only exist in hostile environments. After some years of advanced studies, species of the super phylum Crenarchaeota were noted present in the open sea and the regions of the artic. This expanded the habitat of these domain of life to new areas prompting undertaking of advance elaborate studies and surveys in other similar areas but not limited to harsh areas to areas such as the gastrointestinal tract of animals (Zaremba et al., 2017; Baker et al., 2016; Meng et al., 2014; Rinke et al., 2013; Kozubal et al., 2013). The phyla currently being worked on have advanced to include Bathyarchaeota, Korarchaeota, Nanoarchaeota, Thaumarchaeota, Micrarchaeota, Pacearchaeota, Lokiarchaeota, Thorarcheaota, Woesearcheaeota, Verstraetearchaeota, Odinarchaeota, Hemidallarchaeota, Diapherotrites, Hadesarchaeota and Aigarchaeota well-defined by the functional basis and comparative genomics (Spang et al., 2017; Seitz et al., 2016; Vanwonterghem et al., 2016). This is causing an advancement in the phylogenetic tree and this shows a possibility of more phyla being discovered because of such expansion in due course. Some of these phyla are yet to be unwound as little is currently understood. Recent methodology of studies/survey carried out by scholars and modern sequencing techniques opens up room for such advancement (Spang et al., 2017; Seitz et al., 2016; Vanwonterghem et al., 2016). Other than archaea potential to survive in harsh environments, its distribution is determined by their typical prokaryotic lifetime style fostering their diversification (Compte-Port et al., 2017; Logares et al., 2009). With this availability, it defines whether microbes will be present or absent. Methanogenesis occurs during the gut fermentation and methane gas is released in the final step of biomass degradation from the fermentation chambers (Sollinger et al., 2018). Gut Archaea are prokaryotic microbes that initiates and fuel gut methanogenesis (Stewart et al., 2018). The Archaea critical for methane emissions are methanogens and are found freely in the ruminants' gut in association with other micro-organisms (Stewart et al., 2018; Kusar and Avgustin, 2010). The specific micro-organisms in this mutual association are bacteria, protozoa, fungi, methanogens and bacteriophages. The host in this kind of association, facilitates the microorganisms' physical and chemical requirements (Martin et al., 2010). Some of the byproducts that are generated are released in gaseous forms by ruminants to the atmosphere are greenhouse gases agents and they cause undesired greenhouse effects when released way past the accommodatable levels. These gases are potent and permit excess sunbeams in form of short wave radiations to freely pass through the earth's atmosphere thus elevating global temperatures on the terrestrial and oceanic surfaces to high levels (past $15^{\circ} \mathrm{C}$ ) unfit for biotic existences (Mirzaei-Aghsaghali and Maheri-Sis, 2015). The primary known greenhouses gases are industrial gases such as sulfur hexafluoride, hydro-fluorocarbons and fluorocarbons; Carbon dioxide $\left(\mathrm{CO}_{2}\right)$, Methane $\left(\mathrm{CH}_{4}\right)$, Nitrous oxide $\left(\mathrm{N}_{2} \mathrm{O}\right)$ and ozone $\left(\mathrm{O}_{3}\right)$ and the atmospheric water vapours that equally contributes to the natural greenhouse effect (IPCC, 2007). Methane production from livestock has attracted global attention due to; their input on the greenhouse gas effects, contribution to global warming and negative effects on farmers' productivity (Sustr et al., 2014; Moss et al., 2000). Reducing methane emissions rate is a primary and vital element of environmental control agenda (Giuburunca et al., 2014; Schmidt, 2006). Success of any strategy to reduce livestock methane emission for increased farm production and efficiency heavily relies on understanding the quantity, type and/or distribution of methanogenic species in livestock (Wright et al., 2007). The objective of this study 
was to determine the factors contributing to the methanogens gut distribution in smallholding dairy cows. This study specifically focused on the methanogens from the phylum Euryarchaeota.

\section{Materials and Methods}

\section{Statement of ethics}

This research study was performed under the International Livestock Research Institute (ILRI) and the University of Nairobi (UON), Faculty of Veterinary Medicine Institutional Animal Care and Use Committee (IACUC) accepted procedures and guidelines.

\section{Description of the experimental sites}

This research work was undertaken in one experimental site in Kenya, Kiambu County (Kanyariri, UON veterinary and teaching farm) and other two sites in Tanzania; Rungwe and Lushoto districts. The Tanzanian experimental animals were under the umbrella of Tanzania Dairy Project led by ILRI dubbed "Maziwa Zaidi platform". Kanyariri $\left(1^{\circ} 14^{\prime} 33.4^{\prime \prime} \mathrm{S}, 36^{\circ} 42^{\prime} 36.3^{\prime \prime} \mathrm{E}\right.$ latitude and longitude respectively) is located in Lower Kabete of Kiambu County, Kenya. It experiences a sub-humid condition with a maximum average temperature of $21.1^{\circ} \mathrm{C}$ and a minimum of $17.1^{\circ} \mathrm{C}$ and a maximum rainfall of $246 \mathrm{~mm}$ and its lowest being $17 \mathrm{~mm}$ in a year on rainy seasons and annual average precipitation of between $700 \mathrm{~mm}$ and $2000 \mathrm{~mm}$ (https://vetfarm.uonbi.ac.ke/).

Lushoto district ( $4^{0} 57^{\prime} 54.3168^{\prime \prime}$ S, $38^{0} 30^{\prime} 5.7132^{\prime \prime}$ $\mathrm{E}$ latitude and longitude respectively) is a medium potential agricultural land. Its annual rainfall is between $2000 \mathrm{~mm}$ and $500 \mathrm{~mm}$ : with March to June being the longest rainy season. It has around 29, 200 crossbreds (Mfune, 2015).

Rungwe (latitude $9^{\circ} 15^{\prime} 00^{\prime \prime} \mathrm{S}$ and longitude $33^{\circ}$ $40^{\prime} 00^{\prime \prime}$ E) receives rainfall throughout the year with 2700 being high and a low of $900 \mathrm{~mm}$. Its temperature range is $18^{0}-25^{\circ} \mathrm{C}$. The region has about 26,137 crossbreds with an average of 4 per household (Habimana et al., 2018).

The research sites in Tanzania were selected due to the availability of a wide range of cattle breeds, and the sites being emerging high potential dairy regions. However, there is greater emphasis on dairy farming in Rungwe, than Lushoto, with majority of dairy animals being fed under zero grazing conditions (Mwakaje, 2008).

\section{Sample collection from crossbred animals from Tanzania}

Experimental animals were purposefully drawn from the dairy project facilitated by ILRI in Tanzania. This was guided by information kept by the project administrators and willingness of the farmers keeping the dairy animals to participate in the project. Eighteen crossbred dairy cows from each district were purposefully sampled based on their genotypes as illustrated by Cheruiyot et al., (2018).

The collection of fecal samples was done by palpating the rectum using clean sterilized lubricated hand sleeves. About $250 \mathrm{~g}$ of the rectal matter as described by Habimana et al., (2018) was hand grabbed and a proportion was transferred into a sterile $50 \mathrm{ml}$ falcon tube and labeled according to the animal and the area of collection. The samples from each were immediately placed in cool boxes and shipped to Biosciences East and Central Africa (BecA), ILRI, Nairobi. The low temperatures ensured that the samples remained homogenous with no freezethawing effects. This was key for these samples as they awaited methanogenic DNA extraction and subsequent pipeline analysis as recommended by Henderson et al., (2015).

\section{Sample collection from the experimental animals at Kanyariri}

Six mature lactating cows kept at the UON Veterinary farm (Kanyariri) were considered for this study. The animals were chosen based on their health history, lactation phase, body condition score, body weight, lactation stage and availability of their records (both reproductive and production). The six animals were sampled for both the ruminal fluid and the fecal matter. The methodology for collection of fecal samples is as discussed in the Tanzanian samples above, while collection of ruminal fluid was as heighted below.

\section{Ruminal fluid collection}

About $250 \mathrm{ml}$ of rumen fluid was sucked using a flexible stomach tube from each experimental animal with the first batch that was sucked and 
expelled to reduce on chances of extracting saliva. Fluid obtained was separated using a muslin bag and the undesired materials were discarded. About $50 \mathrm{ml}$ of the rumen fluid was retained from each sample and transferred into falcon tubes. The samples obtained were carefully labelled in accordance to the experimental animals that it was withdrawn to avoid any confusion and mismatch. The samples were immediately kept in iceboxes and sent to BecA, ILRI, Nairobi laboratory for microbial DNA analysis.

\section{DNA Extraction and its subsequent analysis}

Before analysis, the ice-covered samples were thawed to room temperature then whirl wound thoroughly at maximum speed for at least 30 seconds as illustrated by Habimana et al., (2018), for homogeneity. Whole genomic DNA was extracted separately from all samples using the QIAamp DNA Mini Stool Kit (Qiagen, USA), adhering to the manufacturer's instructions. The quality of extracted DNA was visualized by $1.2 \%$ Agarose gel electrophoresis and the quantity determined by Nanodrop Spectrophotometry (Nanodrop Technologies). The DNA was stored at $-20^{\circ} \mathrm{C}$ awaiting further analysis procedures.

\section{Illumina sequencing library construction}

Library preparation was achieved using the Nextera DNA Preparation Kit and the Nextera Index Kit (Illumina, San Diego, CA, USA), following the manufacturer's instructions (Nextera and Practices, 2013). This was then followed by Illumina Miseq sequencing. In summary, library preparation involved two limited step PCRs. In the first PCR DNA underwent fragmentation and addition of Nextera Indexes 1 and 2 for identification of specific samples. The second PCR was to amplify this already labelled DNA fragments. After the first PCR, the libraries were cleaned using Zymo DNA extraction kit, while after the second PCR libraries were sized selected using AMPure, XP beads (A63881, Beckam Coulter, Brea, CA, USA). The final library's concentration was assessed using Qubit Assay Kit (Life Technologies Corporation, Grand Island, NY, USA), and their average size measured using the Bioanalyzer tapestation 2200 (Agilent Technologies, Santa Clara, USA). Finally, the paired end sequencing was done using the Illumina Miseq third Version system.

\section{Quality control and characterization of the reads}

For data quality assurance, it was checked using Fast QC/V 0.11.3. Low quality bases (maintaining a length threshold of 100 base pairs and quality of 20) was dynamic trimmed using SolexaQA $++/$ 3.1.3. A second trimming was done (Fast $X$ toolkit- Fast $X$ trimmer) as sacrificing sequences with low quality scores is noted to improve the accuracy of analyses significantly (Yun and Yun, 2014). This was then followed by length sorting for the trimmed-out bases. This was done using SolexaQA ++/3.1.3 at $-\mathrm{d} 20$ and an optimal k-mer of 23 were identified. All the trimmed out paired outputs were assembled together using SPAdes (De novo assembly) against each group.

\section{Methanogens taxonomic characterization}

A detailed unleashing of the methanogens was achieved with Mini-Kraken V. 0.10.5 and Krona Tool V. 2.7. The downloaded file format was narrowed to the Archaea and those related to the domain were only singled out for further analysis. The krona graphs were viewed via the interactive interface at galaxy visualizations (https://usegalaxy.org/.)

\section{Statistical Models}

For estimation of the independent parameters and the dependent variable; the following models in three levels were used. This was achieved using logistic regression for linear and mixed models accomplished using IBM SPSS Statistics Version 28.0.0.0.

$$
\begin{aligned}
& \text { Equation } 1 \\
& \qquad \mathbf{Y}_{i j}=\boldsymbol{u}+\text { Genotype }_{\boldsymbol{i}}+\text { Herd }_{j} \\
& +\boldsymbol{E}_{\boldsymbol{i j}}
\end{aligned}
$$

$\mathrm{Y}_{\mathrm{ij}}=$ The individual methanogenic species, $u=$ The population mean, Genotype $i=$ Random effects due to the genotypic effect ${ }_{i}=R G$ (Norwegian Red X Friesian; NR X G; NR X J), RH (Holstein X NR; NR X H), RZ (NR X Zebu; NR X N'Dama), ZR (Zebu X GR; zebu X NR, Zebu X H), FJ (Friesian X Jersey; JXF) (Cheruiyot et al., 2018), Herd $_{j}=$ Fixed effects due to the geographical 
location differences; Lushoto, Rungwe and $\mathrm{E}_{\mathrm{ij}}=$ residual error Regime.

Equation 2

$$
\begin{aligned}
\mathrm{Y}_{i j k}=u+\text { Genotype }_{i} \\
+ \text { Sample }_{j} \\
+ \text { Cluster }_{k} \\
+E_{i j K}
\end{aligned}
$$

$\mathrm{Y}_{\mathrm{ijk}}=$ The individual methanogenic species (For the Kanyariri herds), $u$ = The population mean,

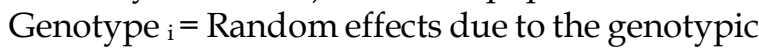
effect, ${ }_{i}=$ FJ (Friesian X Jersey; J X F), F (Friesian) and $\mathrm{J}$ (Jersey), Cluster ${ }_{\mathrm{k}}=$ Fixed effects due to the management segments, $1=$ Low production with low supplementation and $2=$ High intense use of supplements, Sample $_{j}=$ Fixed sample collection sites within the GIT; Fecal and the rumen fluid and $\mathrm{E}_{\mathrm{ijk}}$ residual error.

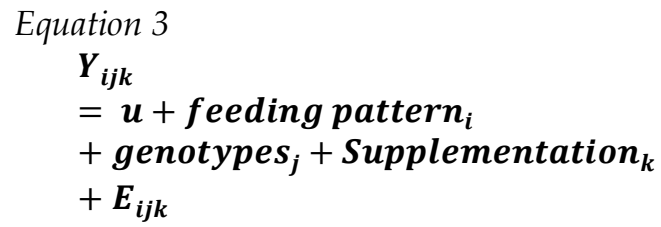

$\mathrm{Y}_{\mathrm{ijk}}=$ Methanogenic species, $u=$ Population mean, Feeding pattern $n_{i}=$ Grazing + Supplementation or Grazing without any supplementation, Genotype ${ }_{j}=$ Random effects due to the genotypic effect, $\mathrm{j}=\mathrm{RG}$ (Norwegian Red X Friesian; NR X G; NR X J), RH (Holstein X NR; NR X H), RZ (NR X Zebu; NR X N'Dama), ZR (Zebu X GR; zebu X NR, Zebu X H), FJ (Friesian X Jersey; J XF), Geographical location regions $=$ Rungwe, Lushoto and UON- Kanyariri and $\mathrm{E}_{\mathrm{ijk}}$ residual error.

\section{Results}

Euryarchaeal methanogens from 12 families were identified during this study (Figure 1).
The most abundant (with the highest number of species noted) family was Methanobacteriaceae with 8 different species followed by the family Methanosarcinaceae and the least was Methanospirillaceae (Figure 1). The respective diversity of species and their abundance were also noted during this study and presented in Table 1 . The factors at play during this study were noted and complied (Table 2).

Effects of feeding regime exposed to dairy cattle on methanogens

Some methanogens (11 species) were only noted from areas where dairy cows were grazed and supplemented with concentrates during the time of the study. These species were: Candidatus Methanomassiliicoccus intestinalis, Methanocella conradii, Methanocella paludicola, Methanoculleus marisnigri, Methanotorris igneus, Methanosalsum zhilinae, Methanolobus psychrophilus, Methanosaeta concilii, Methanococcoides burtonii, Methanothermobacter marburgensis and Methanosphaerula palustris as illustrated in Table 1. The following 5 species of methanogens were only noted from areas where grazing with no supplementation was done: Methanobacterium sp. MB1, Methanococcus vannielii, Methanosarcina acetivorans, Methanospirillum hungatei and Methanocaldococcus sp. Fs 406- 22 as illustrated in Table 1 . The other species that were noted during this study were either present in one of the areas where the study was carried out and/or absent in the other as shown in Table 1. This made it impossible to reliably attribute their presence and distribution on feeding that was practiced. Upon statistical analysis, the feeding regime exposed to the cross bred dairy cattle were statistically significant at the $P$ value $<0.1$ as shown in Table 2. Feeding that was practiced in the area of study contributed to determination of the presence of methanogens. 


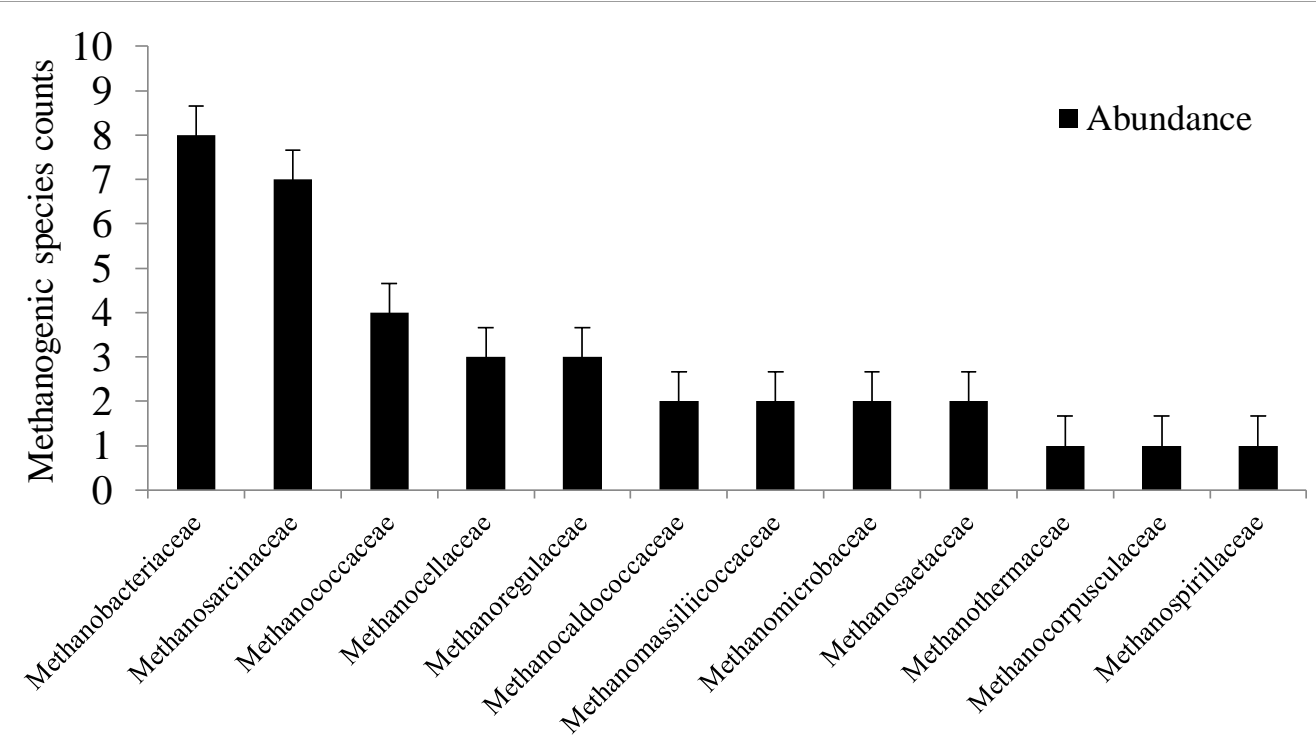

Methanogenic families

Figure 1. A Chart Showing the Methanogenic Families Abundance from the Study Sites.

Table 1. Methanogenic Species Presence, Distribution and Abundance from the Study Area

\begin{tabular}{llllll}
\hline Methanogenic Species & Rumen & \multicolumn{2}{l}{ Fecal } & \multicolumn{2}{l}{ Total } \\
\cline { 2 - 5 } & UON & UON & Lushoto & Rungwe \\
\cline { 2 - 4 } Methanobrevibacter ruminantium & 6 & 5 & 9 & 13 & 33 \\
Methanobrevibacter smithii & 6 & 3 & 6 & 12 & 27 \\
Methanocorpusculum labreanum & 6 & 1 & 4 & 4 & 15 \\
Methanobrevibacter sp. AbM4 & 4 & 1 & 5 & 3 & 13 \\
Methanosaeta harundinacea & 4 & 4 & 2 & 3 & 13 \\
Candidatus Methanomethylophilus alvus & 3 & 6 & - & - & 9 \\
Methanococcus maripaludis & 3 & - & - & 4 & 7 \\
Methanococcus voltae & - & 2 & 1 & 3 & 6 \\
Methanoplanus petrolearius & 1 & 1 & 3 & - & 5 \\
Methanococcus aeolicus & 2 & 2 & 1 & - & 5 \\
Methanocella arvoryzae & - & 2 & 1 & 2 & 5 \\
Methanosarcina mazei & 1 & - & 1 & 2 & 4 \\
Methanothermococcus okinawensis & 2 & - & 1 & 1 & 4 \\
Methanosphaera stadtmanae & 2 & 1 & 1 & - & 4 \\
Candidatus Methanomassiliicoccus intestinalis & 1 & 3 & - & - & 4 \\
Methanocella conradii & 2 & 1 & - & - & 3 \\
Methanomethylovorans hollandica & 1 & - & 2 & - & 3 \\
Methanobacterium paludis & - & 1 & 1 & 1 & 3 \\
Methanoregula boonei & - & 2 & 1 & - & 3 \\
Methanocella paludicola & - & 3 & - & - & 3
\end{tabular}




\begin{tabular}{llllll} 
Methanoregula formicica & - & 1 & 1 & 1 & 3 \\
Methanoculleus marisnigri & - & 3 & - & - & 3 \\
Methanosarcina barkeri & 1 & - & 1 & - & 2 \\
Methanothermobacter thermautotrophicus & 1 & - & - & 1 & 2 \\
Methanothermus fervidus & - & 1 & - & 1 & 2 \\
Methanotorris igneus & - & 2 & - & - & 2 \\
Methanobacterium sp. MB1 & - & - & 2 & - & 2 \\
Methanosalsum zhilinae & 1 & - & - & - & 1 \\
Methanolobus psychrophilus & 1 & - & - & - & 1 \\
Methanosaeta concilii & 1 & - & - & - & 1 \\
Methanococcoides burtonii & 1 & - & - & - & 1 \\
Methanothermobacter marburgensis & - & 1 & - & - & 1 \\
Methanosphaerula palustris & - & 1 & - & - & 1 \\
Methanococcus vannielii & - & - & 1 & - & 1 \\
Methanosarcina acetivorans & - & - & 1 & - & 1 \\
Methanospirillum hungatei & - & - & - & 1 & 1 \\
Methanocaldococcus sp. Fs 406- 22 & - & - & - & 1 & 1 \\
\hline Total Abundance & 50 & 47 & 45 & 53 & 195 \\
\hline
\end{tabular}

- Means absences of the species in the said area of study

Table 2. The Interaction Between Different Factors in the Statistical Model on the Presence of Methanogens

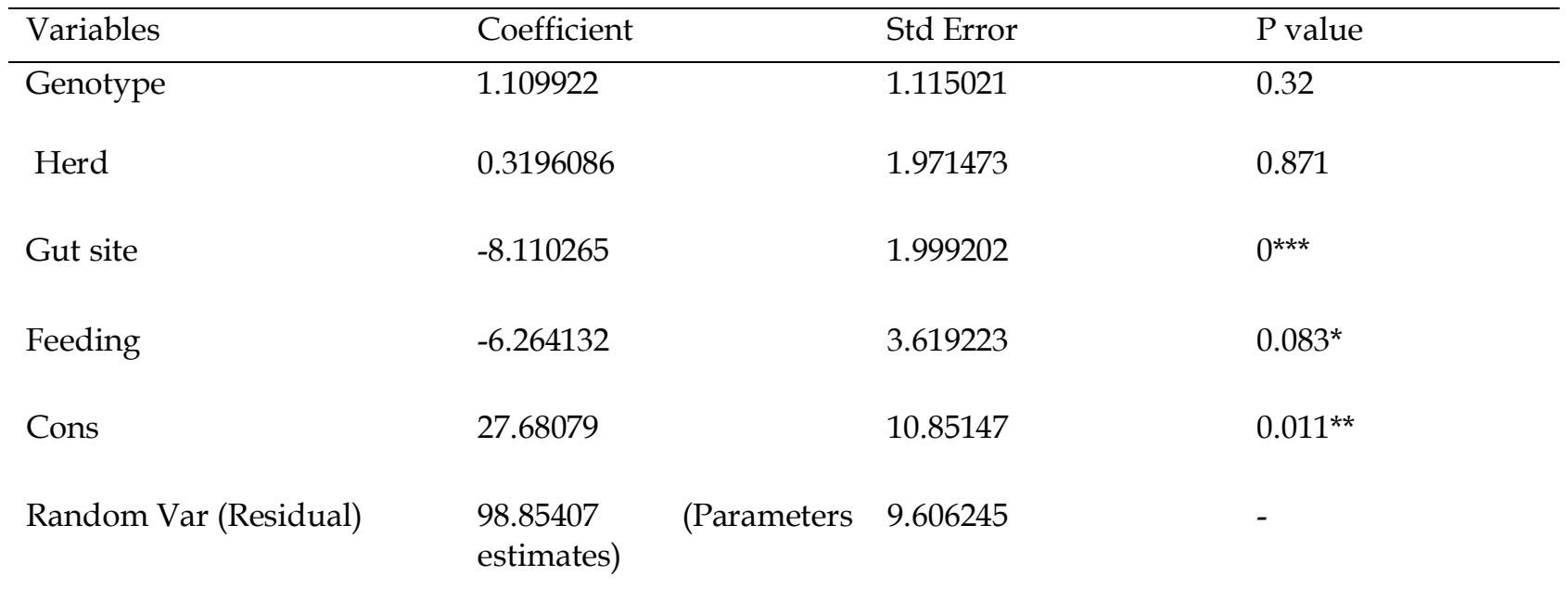

*** Statistically significant at the $\mathrm{P}$ value $<0.01$, ** statistically significant at the $\mathrm{P}$ value $<0.05$ and *statistically significant at the $\mathrm{P}$ value $<0.1$

Effects of the site along the gastro-intestinal tract of dairy cattle on methanogens

A total number of 5 types of species notably were present from the rumen fluid and fecal samples (Table 1). The species were present at the rumen and the rectum and were distributed across all study areas. Their presence was also very abundant when compared to those that were present in either site of the gastrointestinal tract
(Table 1). The following 6 methanogen species were only identified from the rumen fluid: Candidatus Methanomethylophilus alvus, Methanocella conradii, Methanosalsum zhilinae, Methanolobus psychrophilus, Methanosaeta concilii and Methanococcoides burtonii (Table 1). The following two species of methanogens were only present in the fecal samples across all the study areas except for the rumen fluid: 
Methanobacterium paludis and Methanoregula formicica (Table 1). They were noted to occupy the rectum of some of the dairy. Some other methanogens were either found in UON, Lushoto or Rungwe areas and not in any other/all the other study areas in relation to the rumen and the rectum (Table 1). The influence of the factor of the location along the gastrointestinal tract was statistically significant at the $\mathrm{P}$ value $<0.01$ as illustrated by Table 2 . There was no statistical significance of the herd where the dairy cattle were raised and the genotypes of these animals (Table 2). These factors were reportedly not to statistically influence the distribution of methanogens and their presence in the area of study. However, the following 3 methanogens were specific to Kenya: Candidatus Methanomethylophilus alvus, Candidatus Methanomassiliicoccus intestinalis and Methanocella conradii and these 4 were specific to Tanzania; Methanococcus vannielii, Methanosarcina acetivorans, Methanospirillum hungatei and Methanocaldococcus sp. Fs 406- 22 (Table 1).

\section{Discussion}

The rumen of ruminants is an important chamber where fermentation is achieved with the symbiotic relationship between viruses, archaea, fungi, bacteria and protozoa. Archaea forms a proportion of $3 \%$ of these organisms to release among others; microbial proteins, volatile fatty acids, ammonia and methane (Rey et al., 2014). For the non-ruminants, it is noted that large volumes of hydrogen and methane gases are produced from the rectum during partial or full fermentation by anaerobes for the undigested carbohydrates (Trintafylou et al., 2014). This study focused on these two sites (rumen and rectum) of the gastrointestinal tract.

\section{Euryarchaeal Methanogens presence and diversity}

Euryarchaeal methanogens has recorded 155-200 isolated species which are clustered into 4 classes, 7 orders, 14 families and 29-35 diverse genera and are not easily cultured when compared to bacteria ( Singh et al., 2013; Luo et al., 2009). This study recorded the presence of 12 families (Figure 1). This is an illustration of a high representation of the taxonomic families in the study areas. This study registered a high presence of methanogens from the family Methanobacteriaceae (21.6\%) and $8.1 \%$ for Methanocellaceae which is different from what $\mathrm{Zu}$ et al., (2016) had reported of the family Methanocellaceae (also referred to as rice cluster) being abundant (37.3\%) from paddy soils followed by Methanobacteriaceae at $22.1 \%$. In a study by Kim et al., (2014), Methanobacteriaceae was also reported dominant. The difference is deemed to have been brought about because of the difference in ecology and $\mathrm{pH}$ where these studies were done. Methanomassiliicoccales have been noted to drop in abundance when a diet is changed from forage to supplemented grained diet (Zhu et al., 2016) which was confirmed in this study. Cattle exposed to higher forage diets have been noted to release more methane gases when compared to those on less forage diets. The experimental animals in UON were exposed to concentrates when compared to the Tanzanian herd. The Tanzanian herd did not have presence of these 2 species; Candidatus Methanomethylophilus alvus and Candidatus Methanomassiliicoccus intestinalis as their diet was consistently kept to grazing throughout the study period. The term Candidatus denotes that these species are still candidates for taxonomic placement. The presence of these species in the Kenyan herd is attributable to the diet that was exposed to this herd that shifted hence might have caused their identity to be placed in the family Methanomassiliicoccales but could potentially be slotted elsewhere in the taxonomic tree.

Effects of the host feed exposure on methanogen presences

Methanogens in the rumen and some certain species from Clostiridium and Bacteroides would utilize Hydrogen and Carbon dioxide produced in the chamber by other fermentative members and Formic acid and methylamines produced by other organisms as their substrates to finally release methane gas. In the rumen, methanogens would live either on the rumen fluid or when it attaches on feed materials ingested. In the rumen there are ciliated protozoa and fungi that possesses hydrogenosomes which are able to produce hydrogen through oxidizing malate attracting methanogens as endosymbiont facilitating interspecies hydrogen transfer. The conditions of the host dairy animal influence the 
presence of these symbiotic micro-organisms. Therefore, methanogens being one of these microbes, it influences the presence of methanogens along the different regions of the gastrointestinal tract by giving them room to attach and dwell. Bovine calves fed consistently on milk diets have been noted to produce no methane gas (IPPC, 2000). Methanogens level in lambs has been noted occurring in higher proportion when compared to those of other young ruminants and this is noted to change over time in terms of diversity and abundance amidst other components. A developing stomach of the young ruminant offers room for manipulation of the microbiota therein and feed exposed to any newborn ruminant in a way would determine the microbiota to occupy the tract (Zhou et al., 2014). Sections of the lower gut where methanogens can occupy are ileum, jejunum, duodenum, cecum, colon and rectum. Less diversity has been noted in young calves (3-4 weeks old) but a shift to a higher number in calves changing their diet from milk to fibre rich rations (Zhu et al., 2014). In a study by Hook et al., (2010), the authors reported no significant effects of diet on the concentration of methanogens in the samples of hay and high concentrate diets among non-lactating cattle. This study saw a significant effect of diet exposure to the animals in the different study areas. This is attributable to the variance in the methodology used during the study between the two studies. This study used next generation sequencing techniques coupled with bioinformatics tools that are effective when compared to the former technologies of culturing and archaeal 16S rDNA (Song et al., 2011; Hook et al., 2010).

It has been reported from other studies that methanogens found in the rumen of young ruminants at birth and their early life are as a result of mainly contact with dam's skin at birth or when suckling, their birth canal and the immediate environment during cesarean section or the suckled colostrum/milk (Imprinting) (Rey et al., 2014; Di Mauro et al., 2013). In a study by Wang and coworkers, when young ruminants were offered starter feed rich in starch, the number of methanogens rose steadily helping them to colonize the rumen that early. This was because starchy diet facilitates a high release of hydrogen which is one of the substrates for methanogens (Wang et al., 2017).

\section{Effects of the site of the dairy cattle along the gastrointestinal tract}

With an increase in the feed passage rates, methanogenesis can also be shifted to the other parts of the gut (Hook et al., 2010). This study showed significant number of species at the hind gut. Major concerns and attentions have always been intensified with emphasis to the rumen as the major area for methane release with little activity in the lower section of the gastrointestinal tract. The presence of methanogen species at the lower part of the gut is a strong indication that activities leading to methane release from the rectum could be higher than the 13\% (Hook et al., 2010) of the total methane release initially documented. Depending on the availability of the suitable substrate, methanogens will be present within the gastrointestinal tract. With evidence of environmental climate change, it affects availability of particular substrates. Individual methanogens are expected to change, reduced in their dominance or even adapt to such change in order to survive.

Feed offered to the animal has been noted to influence the presence of methanogenic species. Depending on the nature offered, it will affect the composition and type of methanogens that would be active. Dietary manipulation and forestomach controls (Kumar et al., 2014) have been tried to block methane release. However, some of these inhibitory compounds have undesirable effects on exposure to animals making them inappropriate to use on commercial farms (McAllister and Newbold, 2008). Such compounds are Bromochloromethane, 2-bromoethane sulfonate, chloroform and 3nitrooxypropanol compound which deters the methane release by phasing out the last stage of the methanogenesis process (Haisan et al., 2014). The usage of the substance 3-nitrooxypropanol has been shown to decrease methane production in dairy cattle by $30 \%$. Further, its use does not have negative impact either on production of milk or feed ingestion (Hristov et al., 2015) but its ability to decrease methane production is not passed on to their offspring. The use of some of these products have not been successful due to 
microbial adaptation, animal health concerns, food safety and environmental impacts. Furthermore, the use of some of them have been banned in some countries of the world which has limited their use and distribution (Kobayashi et al., 2016). This leaves scientists with an option of breeding for eco-friendly species that can ensure relay of desired genetics to their offspring without affecting any functionality of the dairy cattle.

\section{Conclusion}

Feeding regime exposed to the dairy cattle at their course of feeding is very important. There is a need to watch on their feeding regime in order not to harm the environment at the expenses of just offering feed to the animal. This study further showed that the rumen and rectum in the gut are capable of hosting methanogens. Volumes of methane gas released from these sites

\section{References}

Baker, B.J., Saw, J.H., Lind, A.E., Lazar, C.S., Hinrichs, K.U., Teske, A.P., and Ettema, T.J.G. (2016). Genomic inference of the metabolism of cosmopolitan subsurface Archaea, Hadesarchaea. Nature Microbiology 1: 1-7.

Cheruiyot, E. K., Bett, R. C., Amimo, J. O., and Mujibi, D. F. (2018). Use of a HighDensity SNP Chip to Characterize Breed Composition of Crossbred Dairy Cattle in Tanzania Use of a High-Density SNP Chip to Characterize Breed Composition of Crossbred Dairy Cattle in Tanzania.

Compte-Port, S., Subirats, J., Fillol, M., SànchezMelsió, A., Marcé, R., Rivas-Ruiz, P., ... \& Borrego, C. M. (2017). Abundance and co-distribution of widespread marine archaeal lineages in surface sediments of freshwater water bodies across the Iberian Peninsula. Microbial ecology, 74(4), 776-787.

Di Mauro, A., Neu, J., Riezzo, G., Raimondi, F., Martinelli, D., Francavilla, R., \& Indrio, F., (2013). Gastrointestinal function development and microbiota. Ital J Pediatr

39 , 15 would vary but both are significant. Target for reduced methane release should entirely be to the whole tract and not only to the fore chambers.

\section{Recommendations}

This study recommends advanced research work to be carried out in order to breed and raise cattle that are less methane emitters and find out any possible interspecies presence correlation. Aspects of less methane emission should also be factored when designing breeding goals and programmes for any country or region.

\section{Acknowledgement}

I would like to thank ILRI, Kenya for the opportunity to undertake data analysis at their campus. Much thanks to the farmers who participated in this research work and UON for facilitating the station-based data collection.

https://doi.org/10.1186/1824-7288-39$15 b$

Galaxy Interface. https://usegalaxy.org/. Accessed 19/6/2019.

Giuburunca, M., Criste, A., Constantinescu, R., Raducu, C., Mihaela, G., Cocan, D., and Miresan, V. (2014). Methane Production in the Rumen and its Influence on Global Warming. https://www.researchgate.net/publicat ion/323258813

Habimana, V., Bett, R.C., Amimo, J. O, Kibegwa, F.M., Githae, D. and Junga, J.O. (2018). Metagenomic analysis of enteric bacterial pathogens affecting the performance of dairy cows in smallholder productions systems. African Journal of Microbiology Research,12(17), 387-398. https://doi.org/10.5897/AJMR2018.884 9

Haisan, J., Sun, Y., Guan, L.L., Beauchemin, K.A., Iwaasa, A., Duval, S., Barreda, D.R., Oba, M., (2014). The effects of feeding 3nitrooxypropanol on methane emissions and productivity of Holstein cows in mid lactation.J.Dairy Sci.97: 3110-319.

Henderson, G., Cox, F., Ganesh, S., Jonker, A., Young, W., Janssen, P. H., and Zunino, P. (2015). Rumen microbial community composition varies with diet and host, 
but a core microbiome is found across a wide geographical range. Scientific Reports.

https://doi.org/10.1038/srep14567

Hook, S. E., Wright, A. D. G., and McBride, B. W. (2010). Methanogens: Methane producers of the rumen and mitigation strategies. Archaea. https://doi.org/10.1155/2010/945785

Hristov, A.N., Giallongo, F.J., Frederick, W.T., Harper, T.M., Weeks, L.H., Branco, F.A., Moate, P.J., Deighton, M.H., Richard, O.S., Kindermann, M.W., and Duval, S. (2015). An inhibitor persistently decreased enteric methane emission from dairy cows with no negative effect on milk production. PNAS. 112 (34) 10663-10668; https://doi.org/10.1073/pnas.15041241 12

IPCC. (2007). Climate Change 2007: impacts, adaptation and vulnerability: Contribution of Working Group II to the fourth assessment report of the Intergovernmental Panel. Genebra, Suiça. https://doi.org/10.1256/0043165023205 17344

Kim, S.Y., Pramanik, P., Bodelier, P.LE., and Kim, P.J. (2014). Cattle manure enhances methanogens diversity and methane emissions compared to swine manure under rice paddy. PLOS ONE 9:e113593.

Kobayashi, Y., Oh, S., Myint, H., and Koike, S. (2016). Use of Asian selected agricultural byproducts to modulate rumen microbes and fermentation. Journal of Animal Science and Biotechnology. https://doi.org/10.1186/s40104-0160126-4

Kozubal, M.A., Romine, M., Jennings, R.D., Jay, Z.J., Tringe, S.G., Rusch, D.B., et al.

(2013). Geoarchaeota: A new candidate phylum in the Archaea from high temperature acidic iron mats in Yellowstone National Park. ISME Journal 7: 622-634.

Kumar, S., Dagar, S. S., and Puniya, A. K. (2012). Isolation and characterization of methanogens from rumen of Murrah buffalo. Annals of Microbiology. https://doi.org/10.1007/s13213-0110268-8
Kusar, D., and Avgustin, G. (2010). Molecular profiling and identification of methanogenic archaeal species from rabbit caecum. FEMS Microbiology Ecology. https://doi.org/10.1111/j.15746941.2010.00980.x

Liu, K., Xu, Q., Wang, L., Wang, J., Guo, W., and Zhou, M. (2017). The impact of diet on the composition and relative abundance of rumen microbes in goat, 30 (4): 531537. Asian Australasian Journal of Animal Sciences. DOI: 10.5713/ajas.16.0353

Logares, R., Bråte, J., Bertilsson, S., Clasen, J.L., Shalchian-Tabrizi, K., and Rengefors, K. (2009). Infrequent marine-freshwater transitions in the microbial world. Curr Trends Microbiol17:414-422

Luo, H., Sun, Z., Arndt, W., Shi, J., Friedman, R., and Tang, J. (2009). Gene order phylogeny and the evolution of methanogens. PLoS ONE. https://doi.org/10.1371/journal.pone.0 006069

Martin, C., Morgavi, D. P., and Doreau, M. (2010). Methane mitigation in ruminants: From microbe to the farm scale. Animal. https://doi.org/10.1017/S175173110999 0620

Mcallister, T., and Newbold, C.J. (2008). Rumen fermentation to reduce methanogenesis. Australian Journal of Experimental Agriculture 48(2).

Meng, J., Xu, J., Qin, D., He, Y., Xiao, X., and Wang, F. (2014). Genetic and functional properties of uncultivated MCG archaea assessed by metagenome and gene expression analyses. The ISME journal 8: 650-9

Mfune, R. L. (2015). Epidemiological study of bovine brucellosis in smallholder dairy cattle in Lushoto and Rungwe districts, Tanzania. MSc.Thesis, 38-39.

Mirzaei-Aghsaghali, A., \& Maheri-Sis, N. (2015). Factors affecting mitigation of methane emission from ruminants: Microbiology and biotechnology strategies. Journal of Animal Behaviour and Biometeorology. https://doi.org/10.14269/23181265/jabb.v4n1p22-31

Moss, A., Jouany, J., Newbold, J., Moss, A., Jouany, J., and Newbold, J. (2000). 
Methane production by ruminants: its contribution to global warming: HAL Id : hal-00889894 Review article Methane production by ruminants: its contribution to global warming, Biology, Environmental Science.

Mwakaje, A. G. (2008). Dairy farming and biogas use in Rungwe district, South-west Tanzania: a study of opportunities and constraints. Renewable and Sustain. Energy Rev. 12, 2240-2252.

Nextera, E., and Practices, B. (2013). Nextera DNA sample kit. Journal of Energy. 5(800):1-22

Patra, A., Park, T., Kim, M., \& Yu Z. (2017).

Rumen methanogens and mitigation of methane emission by anti-methanogenic compounds and substances. J Animal Sci Biotechnol 8, 13

https://doi.org/10.1186/s40104-0170145-9

Rey M., Enjalbert F., Combes S., Cauquil L., Bouchez O., \& Monteils V., (2014). Establishment of ruminal bacterial community in dairy calves from birth to weaning is sequential. J. Appl. Microbiol. 116:245-257. doi:10.1111/jam.12405.

Rinke, C., Schwientek, P., Sczyrba, A., Ivanova, N.N., Anderson, I.J., Cheng, J.-F., et al. (2013). Insights into the phylogeny and coding potential of microbial dark matter. 151. Nature 499: 431-437.

Schmidt, T. M. (2006). The maturing of microbial ecology. International Microbiology.

Seitz, K.W., Lazar, C.S., Hinrichs, K.U., Teske, A.P., \& Baker, B.J. (2016). Genomic reconstruction of a novel, deeply branched sediment archaeal phylum with pathways for acetogenesis and sulfur reduction. ISME Journal 10: 16961705

Singh, K. M., Tripathi, A. K., Pandya, P. R., Parnerkar, S., Kothari, R. K., \& Joshi, C. G. (2013). Molecular Genetic Diversity and Quantitation of Methanogen in Ruminal Fluid of Buffalo (Bubalus bubalis) Fed Ration (Wheat Straw and Concentrate Mixture Diet).

Genetics Research International, 7. https://doi.org/10.1155/2013/980191
Sollinger, A., Tveit, T., Poulsen, M., Noel, J., Bengtsson, M., Bernhardt, J., \& Højberg, O. (2018). Holistic Assessment of Rumen Microbiome Dynamics through Quantitative Metatranscriptomics Reveals Multifunctional Redundancy during Key Steps of Anaerobic Feed Degradation, American Society for Microbiology, 3(4), 1-19.

Song, K.M., Li, Z.X., Oh, K.Y., Lee, C., \& Hyun Y. (2011). Control of Methane Emission in Ruminants and Industrial Application of Biogas from Livestock Manure in Korea. Asian-Aust. J. Anim. Sci. Vol. 24, No. 1: 130 - 136

Spang, A., Caceres, E.F., \& Ettema, T.J.G. (2017). Genomic exploration of the diversity, ecology, and evolution of the archaeal domain of life. Science 357

Stewart, R. D., Auffret, M. D., Warr, A., Wiser, A. H., Press, M. O., Langford, K. W., and Watson, M. (2018). Assembly of 913 microbial genomes from metagenomic sequencing of the cow rumen. Nature Communications. https://doi.org/10.1038/s41467-01803317-6

Sustr, V., Chronakova, A., Semanova, S., Tajovsky, K., and Simek, M. (2014). Methane production and methanogenic archaea in the digestive tracts of millipedes (Diplopoda). PLOS ONE. https://doi.org/10.1371/journal.pone.0 102659

Triantafyllou, K., Chang, C., \& Pimentel, M. (2014). Methanogens, methane and gastrointestinal motility. Journal of neurogastroenterology and motility, 20(1), 31-40.

https://doi.org/10.5056/jnm.2014.20.1. 31

University of Nairobi Website, Department of Vet farm. Accessed 28/7/2021. https://vetfarm.uonbi.ac.ke/

Vanwonterghem, I., Evans, P.N., Parks, D.H., Jensen, P.D., Woodcroft, B.J., Hugenholtz, P., \& Tyson, G.W. (2016). Methylotrophic methanogenesis discovered in the archaeal phylum Verstraetearchaeota. Nature Microbiology 1:1-9. 
Wang, Z., Elekwachi, C. O., Jiao, J., Wang, M., Tang, S., Zhou, C., Tan, Z., \& Forster, R. J. (2017). Investigation and manipulation of metabolically active methanogen community composition during rumen development in black goats. Scientific reports, $7(1), \quad 422$. https://doi.org/10.1038/s41598-01700500-5

Wright, A.-D. G., Auckland, C. H., \& Lynn, D. H. (2007). Molecular Diversity of Methanogens in Feedlot Cattle from Ontario and Prince Edward Island, Canada. Applied and

Environmental Microbiology. https://doi.org/10.1128/AEM.00103-07

Yun, S., \& Yun, S. (2014). Masking as an effective quality control method for nextgeneration sequencing data analysis. BMC Bioinformatics.

Zaremba-Niedzwiedzka, K., Caceres, E.F., Saw, J.H., Bäckström, Di., Juzokaite, L., Vancaester, E., et al. (2017). Asgard archaea illuminate the origin of eukaryotic cellular complexity. Nature 541: 353-358

Zhou, M., Chen, Y., Griebel, P. J., \& Guan, 1. (2014). Methanogen prevalence throughout the gastrointestinal tract of pre-weaned dairy calves. Gut microbes, 5(5), 628-638.

https://doi.org/10.4161/19490976.2014. 969649

Zhu, Z., Løvendahl, P., Poulsen, M., Samantha, P., and Noel, J. (2016). Dynamics of rumen bacterial and archaeal communities in dairy cows over different lactation cycle stages.

Zu, Q., Zhong, L., Deng, Y., Shi, Y., Wang, B., Jia, Z., Lin, X., and Feng, Y. (2016). Geographical distribution of methanogenic archaea in nine representative paddy soils in China. Front Microbiol 